\title{
Genetic Programming Algorithm for Designing of Control Systems
}

\section{Genetic Programming Algorithm for Designing of Control Systems}

\section{Krystian Łapa, Krzysztof Cpałka, Andrzej Przybył}

Institute of Computational Intelligence; Częstochowa University of Technology; Al. Armii Krajowej 36, 42-200 Częstochowa, Poland; phone: +48343250546; fax: +48343250546; e mails: \{krystian.lapa,krzysztof.cpalka,andrzej.przybyl\}@iisi.pcz.pl

Corresponding author: krzysztof.cpalka@iisi.pcz.pl

Genetic programming algorithms and other population-based methods are a convenient tool for solving complex interdisciplinary problems. Their characteristic feature is that they flexibly adapt to the problem and expectations of a designer. In this paper, they are used for designing complex control systems. In particular, a new approach for automatic designing of PID-based controllers which are resistant to noises in the measuring path is proposed. It is based on the knowledge about an object model and capabilities of the genetic algorithm and genetic programming. Not only do these make it possible to tune parameters and select the structure of PID-based controllers, but they also tune parameters of some additional components of a complex controller structure, like parameters of finite impulse response (FIR) filters. The idea of the proposed approach relies on proper encoding of the controller and a dedicated way of evolutionary processing of encoded solutions. The approach proposed in this paper has been tested using a typical control problem, i.e. a DC motor control problem. KEYWORDS: artificial intelligence, controller, PID, selection of structure, selection of parameters, genetic programming.

\section{Introduction}

In this paper, a few possibilities of using population-based algorithms in control systems are presented. Moreover, a new dedicated variation of genetic programming (GP) for designing of control systems is proposed. This method makes it possible to design systems that can adapt to a given problem and expectations with which a designer is faced.

Controllers play an important role in control systems. Their aim is to influence the control object in such a way so as to make that object operate in the way it 
is expected to. In the literature many types of control methods can be found including: adaptive control [37], fuzzy control [12, 31], neuro-fuzzy control [29], fuzzy-integral-sliding [50], type-2 fuzzy control [48], internal model control [33], model predictive control [41], neural network control [20], nonlinear feedback linearization control [32], nonlinear optimal control [18], sliding mode control [24], etc. Although new types of controllers are still being sought for, controllers based on correction terms (proportional-integral-derivative-based controllers) still have an important place in control problems. A number of simple PID controllers can be interconnected to form a more complex controller structure, e.g. a cascade structure. Such controllers are considered in this paper and will be referred from now on as PIDCs. Such controllers have a clear structure (the function of the correcting elements is easily interpretable), work well in most control systems [36], mostly have practical applications [39], and have many simple methods for tuning theirs parameters (in the case of a strictly defined structure) $[5,52]$. Among the methods used for parameter tuning, computational intelligence based methods (population-based algorithms in particular) take an important place in the literature [9, $14,42,47,51]$. This group of methods also includes genetic programming $[15,16,25,35,46]$. The GP is based on processing of the population of individuals (by using evolutionary operators) and its evaluation. Ultimately, the GP algorithm selects from the population an individual that best suits the defined evaluation criteria for the problem under consideration. Each individual from the population represents a single solution (e.g. one PIDC) which is encoded in the form of a tree that consists of nodes and leaves. In the tree each leaf has an assigned value (e.g. a numeric value or index of controller input signal) and each node has an assigned mathematic operator for calculating the values of nodes and leaves attached under it.

Most typical controller designing methods work on rigidly defined PIDC structures. What distinguishes the GP is the capabilities of producing dynamic structures in the form of a tree. The choice of proper structure could prove to be a significant problem (despite the indications given in the literature), especially for

\section{Table 1}

The main properties of controller designing methods

\begin{tabular}{l|c|c|c|c|c|c|c|c}
\multicolumn{1}{|c|}{ method } & f1 & f2 & $\mathbf{f 3}$ & $\mathbf{f 4}$ & $\mathbf{f 5}$ & $\mathbf{f 6}$ & $\mathbf{f}$ f & $\mathbf{f 8}$ \\
\hline Agharkakli et al. [3] & no & no & no & no & yes & yes & no & no \\
\hline Cheon et al. [11] & yes & no & no & no & no & no & no & no \\
\hline Choi et al. [13] & yes & no & no & no & yes & no & yes & no \\
\hline Duan et al. [19] & no & no & no & no & no & no & yes & no \\
\hline Gil et al. [22] & no & no & no & no & no & no & yes & no \\
\hline Ko and Wu [26] & yes & no & no & no & yes & no & no & no \\
\hline Lai et al. [27] & no & no & no & no & yes & no & no & no \\
\hline Eapa and Cpałka [28] & yes & yes & no & yes & yes & yes & yes & yes \\
\hline Eapa and Cpałka [29] & yes & yes & yes & yes & yes & yes & yes & no \\
\hline Łapa et al. [30] & yes & yes & yes & yes & yes & no & yes & no \\
\hline Petkovic et al. [40] & no & no & no & no & no & no & yes & no \\
\hline proposed method & yes & yes & yes & yes & yes & yes & yes & yes \\
\hline
\end{tabular}

f1 -Is a nature-inspired population-based algorithm used? f2 - Can the controller structure be dynamically selected? f3 -Are signal filters used (and signals noise)? f4 -Are the oscillations of the control signal minimized? f5 -Are any specific criteria of the considered problem included and minimized (e.g. over- shooting)? $\mathbf{f} 6$-Is the method verified on different signals than those used during the controller design? fy -Are the presented results 
unconventional applications, and it is usually done by the trial and error method. This demonstrates the GP's advantage over the traditional methods. Nonetheless, the GP cannot be used directly for designing PID-based controllers, but it can be used for the selection of a structure and parameters of tree structures (computer programs) that can be used as controllers. In order to create a specific type of a controller (e.g. a PIDC), the GP must be modified accordingly.

In paper [15], we considered a concept of a PIDC whose structure and parameters could be adjusted automatically using the proposed modification of the GP algorithm. The results obtained and presented in the paper were promising. In this paper, we also consider the problem of choosing the structure and tuning parameters of the PIDC (Fig. 2) on the basis of the model of a control object (Fig. 1). The control system considered in this paper (Fig.1) works in three phases, i.e.: learning, testing, and verification. In the learning phase (which includes the selection of a structure and tuning parameters) a model of the con- trolled object is used. If a satisfactory PIDC is found in this phase (in terms of the assumed evaluation criteria), then the learning can be stopped. The PIDCs designed and optimized in the learning phase are then tested under conditions similar to those in the learning phase (the testing phase) and verified (the verification phase) under conditions other than those in the learning phase (including using a different test signal). After a positive completion of these phases (learning, testing and verification) an implementation on the target hardware platform can be done. The goal of the proposed approach is to choose a simple structure and parameters of the PIDC that meets the accepted evaluation criteria for the problem under consideration. The elements of a novelty presented in this paper are as follows:

- The PIDC structure includes a number of control blocks (CB, Fig. 3) (each composed of a few programmable switches and a single PID controller) and operation blocks (OP, Fig. 4) (each of them includes of a number of programmable switches and a single finite impulse response filter). The proposed genetic programming automatically tunes parameters of these PID controllers and filters (i.e. their order and cut-off frequency) as well as the states of their programmable switches. Such approach shows great possibilities of using genetic programming combined with the genetic algorithm in the design of automatic control systems (both controllers and their supporting elements).

- A new way of designing a PIDC structure: an aggregating operator (OP, Fig. 4) is introduced. Thus, we show that the GP algorithm can be very easily adapted to solve unusual optimization problems.

- The way in which the population is processed by the GP algorithm is improved in comparison to [15]. In addition, the way in which the evolutionary operators are used to search the solution space is updated by introducing intensity of evolutionary operators. This shows that the GP is a flexible algorithm that can be extended by using unconventional evolutionary operators (often adjusted to the problem).

_ A new procedure of initial population initialization is proposed. We have specifically simplified the way of generating a population in comparison to [15].

- A new way of aggregating of the components of the evaluation function is applied. This method was introduced to evaluate the population of individuals in the evolutionary algorithm [29], but it has not been used yet when combined with the algorithm of genetic programming. Thus, we show that the GP algorithm can be easily adapted to solve practical problems in the field of multicriteria (multi-objective) optimization.

The elements of novelty presented in this paper and those considered in the context of control theory and the problem of controller design have been included in Table 1.

The structure of this paper is as follows: in Section 2, the proposed structure is described. Section 3 presents the proposed GP algorithm. Section 4 includes simulation results, and the conclusions are drawn in Section 5.

\section{Figure 1}

The control system (CS) considered in this paper

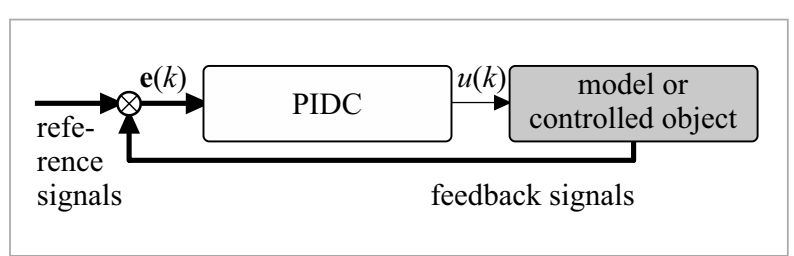




\section{PIDC Structure and Its Encoding for the GP Algorithm}

In this paper a control system (CS, Fig. 1) based on a PIDC (Fig. 2) is considered. The PIDC structure had to be designed in such a way so that it could be selected using the GP algorithm. Therefore, aggregating blocks (OP, Fig. 4) and control blocks (CB, Fig. 3) were separated. CBs are in practice single PID controllers. The $\mathrm{OP}+\mathrm{CB}$ combinations called a node (see Fig. 2). Such approach shows that the way of aggregation of signals in the GP does not have to rely on basic operators (e.g. basic mathematical operators), but it can be more complex and can have its own interpretation.

\subsection{Control Blocks (CB)}

The control blocks perform typical PID activities: proportional (P element, Fig. 3), integral (I element, Fig. 3) and differential (D element, Fig. 3) the input signal. The output signal from the CB can be described as follows:

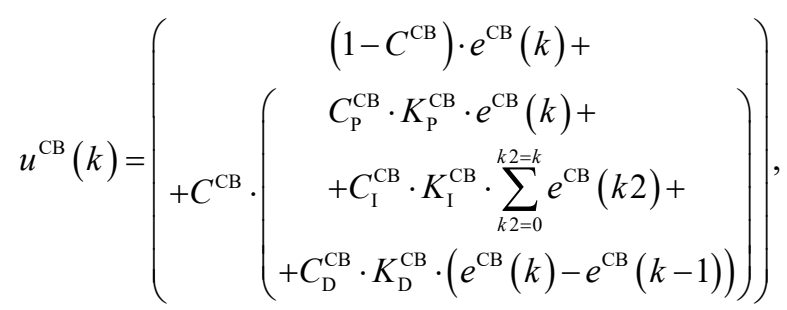

where $e^{\mathrm{CB}}(k)$ stands for $\mathrm{CB}$ input signal (see Fig. 3); $u^{\mathrm{CB}}(k)$ stands for $\mathrm{CB}$ output signal; $K_{\mathrm{P}}^{\mathrm{CB}}$ is the enforcement parameter of P element; $K_{\mathrm{I}}^{\mathrm{CB}}=T_{\mathrm{S}} / T_{\mathrm{I}}\left(T_{\mathrm{I}}\right.$ stands for the integral time constant, and $T_{\mathrm{S}}$-for the control time step); $K_{\mathrm{D}}^{\mathrm{CB}}=T_{\mathrm{D}} / T_{\mathrm{S}}\left(T_{\mathrm{D}}\right.$ stands for the differential time constant); $C_{\mathrm{P}}^{\mathrm{CB}} \in\{0,1\}$ decides on activation of $\mathrm{P}$ term (P term is active if $C_{\mathrm{P}}^{\mathrm{CB}}=1$, see Fig. 3); $C_{\mathrm{I}}^{\mathrm{CB}} \in\{0,1\}$ decides on activation of I term; $C_{\mathrm{D}}^{\mathrm{CB}} \in\{0,1\}$ decides on activation of $\mathrm{D}$ term; $C^{\mathrm{CB}} \in\{0,1\}$ decides on activation of the $\mathrm{CB}$ (CB is active if $C^{\mathrm{CB}}=1$, otherwise $u^{\mathrm{CB}}(k)=e^{\mathrm{CB}}(k)$, see Fig. 3). Parameters $K_{\mathrm{P}}^{\mathrm{CB}}$, $K_{\mathrm{I}}^{\mathrm{CB}}, K_{\mathrm{D}}^{\mathrm{CB}}, C_{\mathrm{P}}^{\mathrm{CB}}, C_{\mathrm{I}}^{\mathrm{CB}}, C_{\mathrm{D}}^{\mathrm{CB}}, C^{\mathrm{CB}}$ are selected automatically by the GP algorithm.

\subsection{Aggregation Blocks (OP)}

OP blocks aggregate signals from CB blocks or controller input signals e $(k)=\left[e_{0}(k), \ldots, e_{l}(k), \ldots, e_{n-1}(k)\right]$.
Figure 2

The general structure of the PIDC controller considered in this paper

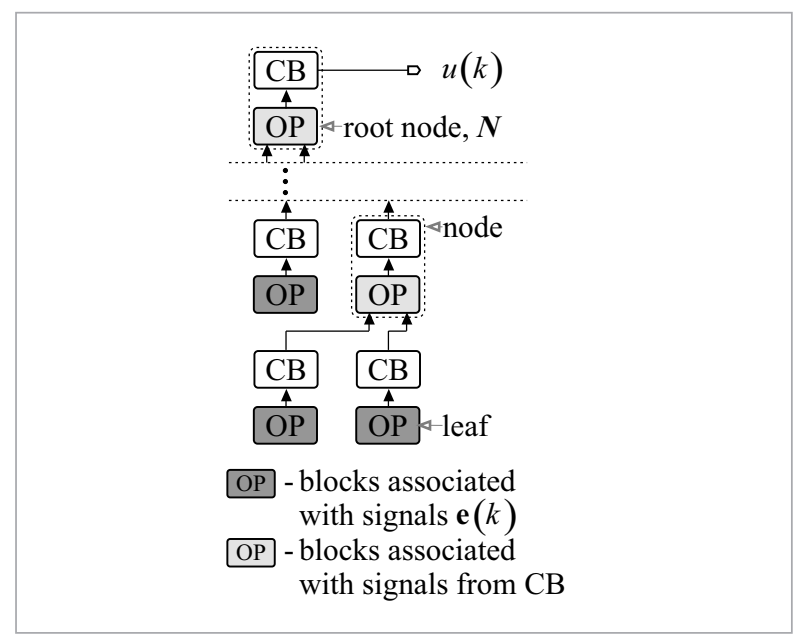

Figure 3

The structure of the control block (CB)

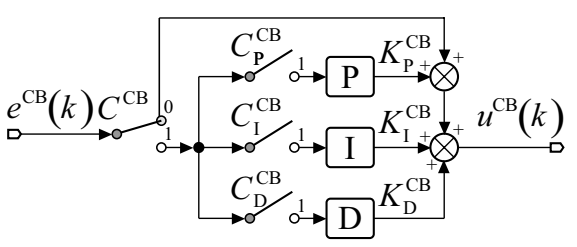

The output signal of the OP blocks is defined as follows:

$$
\begin{gathered}
u^{\mathrm{OP}}(k)=\left\{\begin{array}{l}
\left(u_{\mathrm{L}}^{\mathrm{CB}}(k)+u_{\mathrm{R}}^{\mathrm{CB}}(k)\right) \text { if } l^{\mathrm{OP}}=-1 \text { and } o^{\mathrm{CB}}=0 \\
\left(u_{\mathrm{L}}^{\mathrm{CB}}(k)-u_{\mathrm{R}}^{\mathrm{CB}}(k)\right) \text { if } l^{\mathrm{OP}}=-1 \text { and } o^{\mathrm{CB}}=1 \\
\left(-u_{\mathrm{L}}^{\mathrm{CB}}(k)+u_{\mathrm{R}}^{\mathrm{CB}}(k)\right) \text { if } l^{\mathrm{OP}}=-1 \text { and } o^{\mathrm{CB}}=2 \\
\left(-u_{\mathrm{L}}^{\mathrm{CB}}(k)-u_{\mathrm{R}}^{\mathrm{CB}}(k)\right) \text { if } l^{\mathrm{OP}}=-1 \text { and } o^{\mathrm{CB}}=3 \\
e_{l=l^{\mathrm{OP}}}^{\mathrm{OP}}(k) \text { if } l^{\mathrm{OP}}>-1 \text { and } C_{l=l^{\mathrm{OP}}}^{\mathrm{FL}}=0 \\
e_{l=l^{\mathrm{OP}}}^{\mathrm{FL}}(k) \text { if } l^{\mathrm{OP}}>-1 \text { and } C_{l=l^{\mathrm{OP}}}^{\mathrm{FL}}=1
\end{array}\right. \\
=\left\{\begin{array}{l}
\left(\begin{array}{c}
\mathrm{sgn}\left(o^{\mathrm{CB}}-1.5\right)+1 \\
(-1)
\end{array} u_{\mathrm{L}}^{\mathrm{CB}}(k)+(-1)^{0^{\mathrm{CB}} \% 2} \cdot u_{\mathrm{R}}^{\mathrm{CB}}(k)\right. \\
\left(\begin{array}{c}
C_{l=l^{\mathrm{OP}}}^{\mathrm{FIL}} \cdot e_{l=l^{\mathrm{PP}}}^{\mathrm{FIL}}(k)+ \\
+\left(1-C_{l=l^{\mathrm{PP}}}^{\mathrm{FL}}\right) \cdot e_{l=l^{\mathrm{OP}}}^{\mathrm{OP}}(k)
\end{array}\right) \text { otherwise, }
\end{array}\right.
\end{gathered}
$$


where $l^{\mathrm{OP}} \in\{-1,0, \ldots, n-1\}$ indicates the OP objective: it can be used for aggregation of signals $u_{\mathrm{L}}^{\mathrm{OP}}(k)$ and $u_{\mathrm{R}}^{\mathrm{OP}}(k)$ from other $\mathrm{CB}\left(l^{\mathrm{OP}}=-1\right.$, see Fig. 4$)$, or to attach the controller input signal $e_{l}(k)$ to PIDC $\left(l^{\mathrm{OP}}>-1\right)$ (see Fig. 4), $o^{\mathrm{OP}}$ decides on aggregation of $u_{\mathrm{L}}^{\mathrm{OP}}(k)$ and $u_{\mathrm{R}}^{\mathrm{OP}}(k)\left(u_{\mathrm{L}}^{\mathrm{OP}}(k)+u_{\mathrm{R}}^{\mathrm{OP}}(k)\right.$ for $o^{\mathrm{OP}}=0$, $u_{\mathrm{L}}^{\mathrm{OP}}(k)-u_{\mathrm{R}}^{\mathrm{OP}}(k)$ for $o^{\mathrm{OP}}=1,-u_{\mathrm{L}}^{\mathrm{OP}}(k)+u_{\mathrm{R}}^{\mathrm{OP}}(k)$ for $o^{\mathrm{OP}}=2$, and $-u_{\mathrm{L}}^{\mathrm{OP}}(k)-u_{\mathrm{R}}^{\mathrm{OP}}(k)$ for $\left.o^{\mathrm{OP}}=3\right) ; \operatorname{sgn}(\cdot)$ stands for the signum function; $\%$ stands for the modulo operator; $e_{l}^{\mathrm{FL}}(k)$ stands for input signal $e_{l}^{\mathrm{OP}}(k)$ after passing through the filter block; $u^{\mathrm{OP}}(k)$ stands for the OP output signal; $C_{l}^{\mathrm{FLL}} \in\{0,1\}$ stands for activation of the FIR for $l^{\mathrm{OP}}>-1$ (the filter is active if $C_{l}^{\mathrm{FIL}}=1$, otherwise $u^{\mathrm{OP}}(k)=e_{l}^{\mathrm{OP}}(k)$ ). The parameters $l^{\mathrm{OP}}, o^{\mathrm{OP}}$, and $C_{l}^{\mathrm{FLL}}=1$ are selected automatically by the GP algorithm. Occurred in Eq. (2) and on Fig. 4 marks $u_{\mathrm{L}}^{\mathrm{OP}}(k)$ and $u_{\mathrm{R}}^{\mathrm{OP}}(k)$ are used to indicate these input signals of the OP block that come from child nodes (L-left, R-right) in case of $l^{\mathrm{OP}}=-1$.

\subsection{Filtration Blocks (FIL)}

The filtration blocks (FIL, Fig. 4) are part of the OP blocks used to filter input signals of the PIDC. For the purpose of this paper finite impulse response (FIR) filters were used. FIRs are one of the most commonly used filters. The purpose of FIR filters is to smoothen signal values. It is achieved by averaging weighted values from consecutive time steps. The output value of the filter depends on the filter length and its weights. Although FIR filters are used to improve the quality of control and to reduce impact of signal noise, they might decrease the controller efficiency if used incorrectly $[4,45]$. This is due to the fact that filtration adds a side-effect-phase delay of the filtered signal.

The output signal of the FIL can be described as follows:

$$
e_{l}^{\mathrm{FLL}}(k)=\sum_{l 2=0}^{s^{\mathrm{EL}}-1} b_{l, l 2}^{\mathrm{FL}} \cdot e_{l}^{\mathrm{OP}}(k-l 2),
$$

where $s_{l}^{\mathrm{FIL}}$ stands for odd length of the filter (the filter has to have a middle element), $e_{l}^{\mathrm{OP}}(k-l 2)$ stands for input signal $l$ from $k-l 2$ time step, $b_{l, l 2}^{\mathrm{FIL}}$ stands for the weight of the signal for $k-l 2$ time step calculated as follows [23]:

$$
b_{l, l 2}^{\mathrm{FL}}=\left\{\begin{array}{l}
2 \cdot f t_{l}^{\mathrm{FL}} \text { if } l 2=0.5 \cdot\left(s_{l}^{\mathrm{FIL}}-1\right) \\
\frac{\sin \left(2 \cdot \pi \cdot f t_{l}^{\mathrm{FL}} \cdot\left(l 2-0.5 \cdot\left(s_{l}^{\mathrm{FL}}-1\right)\right)\right)}{\pi \cdot\left(l 2-0.5 \cdot\left(s_{l}^{\mathrm{FL}}-1\right)\right)} \\
\text { otherwise, }
\end{array}\right.
$$

where $f t_{l}^{\mathrm{FLL}}$ stands for filter frequency. The parameters $s_{l}^{\mathrm{FLL}}$ and $f t_{l}^{\mathrm{FL}}$ are selected automatically by the GP algorithm. It should be emphasized that Eq. (4) describes an exemplary form of $b_{l, l 2}^{\mathrm{FL}}$, considered in [23]. The other approaches are described in e.g. [10, 17, 38, 44].

\section{GP Algorithm Adjusted to the Proposed PIDC Structure}

The proposed GP algorithm can be used for the problems where some structure and structure parameters have to be found (e.g. for problems that can be solved using a neural network or a fuzzy system). Therefore, it has a universal character. The idea presented in this paper combines the genetic algorithm (GA) (used for numeric parameter tuning) and genetic programming (GP) (used for parameter tuning and structure selection). The combination of these methods required a change in the process of evolution and adaptation of the evolutionary operators used to search the space of the problem under consideration. This combination of the algorithms, however, has enabled an effective population processing in which both integer (including binary) and real parameters are used simultaneously.

\section{Figure 4}

The structure of the operation block (OP) and the FIL filters used to filter input signals

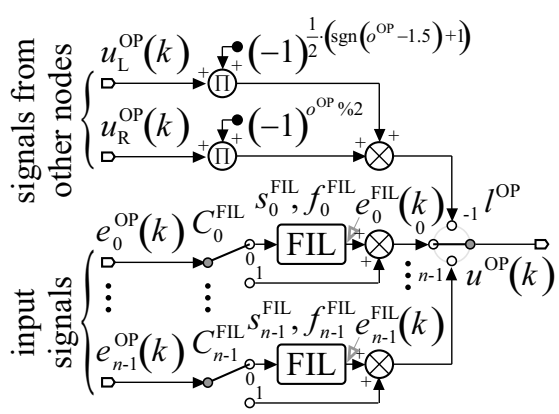


The further part of this section describes: the structure of an individual in the population(Section 3.1), an initialization of the population in the GP algorithm (Section 3.2), evolutionary operators used by the proposed GP algorithm (Section 3.3), a description of the idea of the proposed algorithm (Section 3.4) and a method of evaluation of the individuals in the population (Section 3.5).

\subsection{The Structure of an Individual in the Population}

Each individual of population $\mathbf{X}_{c h}$ is encoded in the form of the tree presented on Fig. 2:

$$
\mathbf{X}_{c h}=\{\mathbf{N}\}=\left\{\begin{array}{c}
K_{\mathrm{P}}^{\mathrm{CB}}, K_{\mathrm{I}}^{\mathrm{CB}}, K_{\mathrm{D}}^{\mathrm{CB}}, \\
C_{\mathrm{P}}^{\mathrm{CB}}, C_{\mathrm{I}}^{\mathrm{CB}}, C_{\mathrm{D}}^{\mathrm{CB}}, C^{\mathrm{CB}}, \\
l^{\mathrm{OP}}, o^{\mathrm{OP}}, C_{l}^{\mathrm{FL}}, \\
u_{\mathrm{L}}^{\mathrm{OP}}, u_{\mathrm{R}}^{\mathrm{OP}}, \\
s_{l}^{\mathrm{FL}}, f_{l}^{\mathrm{FL}}
\end{array}\right\},
$$

where $\mathbf{N}$ is the root of tree (Fig. 2), $u_{\mathrm{L}}^{\mathrm{OP}}$ and $u_{\mathrm{R}}^{\mathrm{OP}}$ are references to child nodes (if they occur - see Fig. 2). Their structure is analogical to node $\mathbf{N}$. A detailed interpretation of the parameters appearing in formula (5) is given in Sections 2.12.3.

The structure (length, complexity) of individual $\mathbf{X}_{c h}$ of the proposed GP is therefore not predetermined, but it can be selected depending on the problem under consideration. It this paper, it is assumed that a tree height cannot be greater than TreeHeight (see Step 6 in Section 3.4).

\subsection{Population Initialization}

Initialization of each individual $\mathbf{N}$ proceeds as follows:

Step 1. Creation of collection $\mathbf{G}$ containing elements of the tree (according to Figs 2-4). It consists of $J$ leaves ( $J$ is a parameter of the algorithm).

Step 2. Random assignment to each $J$ element input signal $e_{i}(k)(i=0,1, \ldots, n-1$, where a positive value indicates that a given element is a leaf) and the rest of the parameters (it takes into account the ranges of values of these parameters which depend on the problem under consideration, including filter parameters).

Step 3. Selecting and removing from collection $\mathbf{G}$ two randomly chosen elements of the tree. If they are associated with the same input signal (parameters $l^{\mathrm{OP}}$ has the same value), then one of parameters $l^{\mathrm{OP}}$ is changed randomly.

Step 4. Creating node $\left(l^{\mathrm{OP}}=-1\right)$ that has the elements selected in Step 3 attached to it. The other parameters of the node are initialized randomly as described in Step 2.

Step 5. Putting the node created in Step 4 in collection $\mathbf{G}$.

Step 6. If collection $\mathbf{G}$ contains two or more elements, go back to Step 3; otherwise go to Step 7 .

Step \%. Selecting from collection $\mathbf{G}$ the last node, and marking it as the root of the tree.

Step 8. Marking the root of the tree (with all nodes and leaves) as a single individual.

The initialization procedure should be repeated for all individuals of the GP population. The created population is then processed according to the procedure described in Section 3.4.

\subsection{GP Evolutionary Operators}

The GP algorithm is dedicated to the PIDC processing and it combines the GA and GP evolutionary operators that have been additionally adjusted to the specification of the problem under consideration. It includes the following operators:

Tuning. This operator processes the real number values of the PIDC $\left(K_{\mathrm{P}}^{\mathrm{CB}}, K_{\mathrm{I}}^{\mathrm{CB}}, K_{\mathrm{D}}^{\mathrm{CB}}, s_{l}^{\mathrm{FIL}}, f_{l}^{\mathrm{FIL}}\right)$. It works analogically to the GA mutation operator. If an assumption that vf stands for the vector of real number values of the PIDC is made, then, the elements of this vector are modified as follows:

$v f_{i}:=v f_{i}+\alpha_{\text {tuning }} \cdot U(-1,1) \cdot\left(v f \mathrm{Max}_{i}-v f \mathrm{Min}_{i}\right)$,

where $\alpha_{\text {tuning }} \in[0,1]$ is an algorithm parameter that stands for tuning intensity; $U(-1,1)$ generates a random number from the range of $[-1,1]$; $\left[v \mathrm{Pin}_{i}, v \mathrm{Max}_{i}\right]$ stands for the ranges of number values $v f_{i}$. This operator modifies only these elements of $\mathbf{v f}$ for which condition $U(0,1)<p_{\text {tuning }}$ is met. The parameter $p_{\text {tuning }} \in(0,1)$ of the GP algorithm is called tuning probability. Because the values generated under Eq. (6) can go beyond the allowable range $\left[v f\right.$ Min $_{i}, v f$ Max $\left._{i}\right]$, the proposed GP algorithm carries 
out the repair procedure described in the next section (Step 6).

Mutation. This operator processes the integer parameters $\left(C_{\mathrm{P}}^{\mathrm{CB}}, C_{\mathrm{I}}^{\mathrm{CB}}, C_{\mathrm{D}}^{\mathrm{CB}}, C^{\mathrm{CB}}, l^{\mathrm{OP}}, o^{\mathrm{OP}}, C_{l}^{\mathrm{FIL}}\right)$. If an assumption that vi stands for the vector of integer number values of the PIDC is made, then, the elements of this vector are modified as follows:

$$
v i_{i}:=U i\left(v_{i M i n}, v_{i M a x}\right),
$$

where $U i\left(v_{i M i n}, v_{i M a x}\right)$ generates a random integer number from the range of $\left[v_{i M i n}, v_{i M a x}\right]$. The operator modifies only these elements of vi for which condition $U(0,1)<p_{\text {mutation }}$ is met. The parameter $p_{\text {mutation }} \in(0,1)$ of the GP algorithm is called mutation probability.

Insertion. This operator selects randomly one node or leaf of the tree (excluding the root node) and replaces it with the root of a new randomly generated tree. The new tree is generated according to the procedure described in Section 3.2 on collection of $J_{\text {insertion }}$ leaves, where $J_{\text {insertion }}<J$. Due to that, the tree does not grow significantly. The insertion is done only if condition: $U(0,1)<p_{\text {insert }}$ is met. The parameter $p_{\text {insert }} \in(0,1)$ of the GP algorithm is called insertion probability.

Pruning. This operator selects randomly one node of the tree (excluding the root node) and replaces it with a randomly generated leaf ( $l^{\mathrm{OP}}$ is set randomly to a value from the range of $[0, n-1])$. The pruning is carried out only if condition $U(0,1)<p_{\text {pruning }}$ is met. Parameter $p_{\text {pruning }} \in(0,1)$ of the GP algorithm is called pruning probability.

Crossover. This operator works on the basis of two PIDCs. From each of them a random node (except the root node) is selected, and the nodes are swapped. The crossover is done only if condition $U(0,1)<p_{\text {crossover }}$ is met. Parameter $p_{\text {crossover }} \in(0,1)$ of the GP algorithm is called crossover probability.

\subsection{Description of the Proposed GP Algorithm}

The GP algorithm is based on a typical schema of a population-based algorithm (see e.g. [2, 42, 43, 49]). It uses two populations, i.e. the main one- $\mathbf{P}$ and an additional one- $\mathbf{P}^{\prime}$ and works according to the following steps:

Step 1. Initialization of population $\mathbf{P}$ with Ninit in-
Table 2

The description of the problem considered in the simulations

\begin{tabular}{l|l}
\hline model: & $\left\{\begin{array}{l}\omega(k+1)=\omega(k)+\frac{T_{s}}{J} \cdot\left(K_{t} \cdot i(k)-b \cdot \omega(k)\right) \\
i(k+1)=i(k)+\frac{T_{s}}{L} \cdot\left(u(k)-R \cdot i(k)-K_{e} \cdot \omega(k)\right)\end{array}\right.$ \\
\hline $\begin{array}{l}\text { parameters of } \\
\text { model: }\end{array}$ & $\begin{array}{l}J=0.01, b=0.10, K_{t}=0.01, \\
R=1.00, L=0.50, K_{e}=0.01\end{array}$ \\
\hline $\begin{array}{l}e_{0}(t), e_{1}(t), e_{2}(t): \\
\text { set signal: }\end{array}$ & $\omega^{*}(t)-\omega(k), \omega(k), i(k)$ \\
\hline $\begin{array}{l}\text { goal of the control } \\
\text { process: }\end{array}$ & $\omega^{*}(t)$ \\
\hline \begin{tabular}{l}
$T_{\mathrm{S}}$, Tsim, nIter $=\frac{T \text { sim }}{T_{\mathrm{S}}}:$ \\
\hline$u$ Min, $u$ Max $:$
\end{tabular} & $0.005 \mathrm{~s}, 5 \mathrm{~s}, 1000$ \\
\hline
\end{tabular}

dividuals $\mathbf{X}_{j}(j=1,2, \ldots$, Ninit $)$ (see Section 3.2. Each individual encodes the whole structure and parameters of the PIDC.

Step 2. Evaluation of population P. Each individual is evaluated according to the criteria of the simulation problem. In the algorithm an assumption that the criteria are minimized is made. The evaluations are calculated on the basis of fitness function $\mathrm{ff}\left(\mathbf{X}_{j}\right)$ (see Section 3.5).

Step 3. Decreasing the size of $\mathbf{P}$ to Npop $<$ Ninit by removing the individuals with worse fitness function values $\mathrm{ff}\left(\mathbf{X}_{j}\right)$.

Step 4. Creating a copy of population $\mathbf{P}$ marked as $\mathbf{P}^{\prime}$. Step 5. Modification of population $\mathbf{P}^{\prime}$. In this step the following operations are performed: tuning, mutation, insertion, pruning, and crossover (see Section 3.3). Because the crossover operator requires two individuals, the second one is drawn from population $\mathbf{P}$.

Step 6. Repair of population $\mathbf{P}^{\prime}$. The repair procedure includes two types of actions. First of all, the values of the real parameters coded in the population are reduced to their permissible ranges. Second, the trees that are taller than TreeHeight are cut to the TreeHeight value (redundant nodes are turned into leaves). The TreeHeight is a parameter of the GP algorithm.

Step \%. Evaluation of $\mathbf{P}^{\prime}$. 
Table 3

The ranges of PIDC parameters adopted in the simulations

\begin{tabular}{l|c|c|c}
\hline parameter & type & min & max \\
\hline$K_{\mathrm{P}}^{\mathrm{CB}}$ & real & 0 & 5000 \\
\hline$K_{\mathrm{I}}^{\mathrm{CB}}$ & real & 0 & 10000 \\
\hline$K_{\mathrm{D}}^{\mathrm{CB}}$ & real & 0 & 10000 \\
\hline$C_{\mathrm{P}}^{\mathrm{CB}}$ & integer & 0 & 1 \\
\hline$C_{\mathrm{I}}^{\mathrm{CB}}$ & integer & 0 & 1 \\
\hline$C_{\mathrm{D}}^{\mathrm{CB}}$ & integer & 0 & 1 \\
\hline$C^{\mathrm{CB}}$ & integer & 0 & 1 \\
\hline$l^{\mathrm{OP}}$ & integer \&-1 & 2 & 1 \\
\hline$o^{\mathrm{OP}}$ & integer & 0 & 3 \\
\hline$f_{l}^{\mathrm{FL}}$ & integer & 0 & 0.5 \\
\hline$s_{l}^{\mathrm{FIL}}$ & real & 0.1 & 10 \\
\hline$C_{l}^{\mathrm{FL}}$ & integer & 2 & 1 \\
\hline
\end{tabular}

Step 8. Creation of a new population $\mathbf{P}$. It combines best Npop individuals (according to the fitness function) from populations $\mathbf{P}$ and $\mathbf{P}^{\prime}$.

Step 9. Correction of the intensity of the GP algorithm. This facilitates moving from exploration to exploitation of the search space and is implemented as follows:

$$
\left\{\begin{array}{l}
p_{\text {tuning }}:=\max \left\{p \operatorname{Min}_{\text {tuning }}, p_{\text {tuning }} \cdot \beta\right\} \\
p_{\text {mutation }}:=\max \left\{p \operatorname{Min}_{\text {mutation }}, p_{\text {mutation }} \cdot \beta\right\} \\
p_{\text {insert }}:=\max \left\{p \operatorname{Min}_{\text {insert }}, p_{\text {insert }} \cdot \beta\right\} \\
p_{\text {pruning }}:=\max \left\{p \operatorname{Min}_{\text {pruning }}, p_{\text {pruning }} \cdot \beta\right\} \\
p_{\text {crossover }}:=\max \left\{p \operatorname{Min}_{\text {crossover }}, p_{\text {crossover }} \cdot \beta\right\},
\end{array}\right.
$$

where $\beta \in(0,1)$ is a reduction factor for the intensity of evolutionary operators.

Step 10. Checking the algorithm stop condition. If the GP algorithm has performed a certain number of steps Nsteps (this is a parameter of the GP algorithm), then, the best individual from population $\mathbf{P}$ is presented and the algorithm stops. Otherwise, the algorithm goes back to Step 4 .

\section{Table 4}

The values of the GP algorithm parameters adopted in the simulations (Nrepeats means the number of performed repetitions of each simulation)

\begin{tabular}{|c|c|}
\hline parameter & value \\
\hline$J$ & {$[5,12]$} \\
\hline$J_{\text {insertion }}$ & {$[3,7]$} \\
\hline TreeHeight & 5 \\
\hline Ninit & 256 \\
\hline Npop & 64 \\
\hline$\alpha_{\text {tuning }}$ & 0.2 \\
\hline$p_{\text {tuning }}$ & 0.8 \\
\hline$p_{\text {mutation }}$ & 0.4 \\
\hline$p_{\text {insert }}$ & 0.2 \\
\hline$p_{\text {pruning }}$ & 0.4 \\
\hline$p_{\text {crosover }}$ & 0.9 \\
\hline$\beta$ & 0.990 \\
\hline pMin tuning & 0.025 \\
\hline pMin $_{\text {mutation }}$ & 0.050 \\
\hline$p$ Min $_{\text {insert }}$ & 0.025 \\
\hline pMin $_{\text {pruning }}$ & 0.050 \\
\hline pMin $_{\text {crosover }}$ & 0.025 \\
\hline Nsteps & 500 \\
\hline Nrepeats & 40 \\
\hline
\end{tabular}

\subsection{Evaluating Individuals}

In the GP algorithm each individual $\mathbf{X}_{c h}$ $(c h=1,2, \ldots, n P o p)$ is evaluated by the fitness function. The goal of the GP algorithm assumed in this paper is to minimize the value of this function. The evaluation function can aggregate many different criteria that are dependent on the problem and user expectations. Their number will be further marked as 
$n F F$. In this paper the criteria used for aggregation are normalized to the unit interval. The fitness function is defined as follows:

$$
\mathrm{ff}\left(\mathbf{X}_{c h}\right)=T_{f=1}^{n F F}\left\{\operatorname{sig}\left(\begin{array}{c}
\mathrm{ff}_{f}\left(\mathbf{X}_{c h}\right), \\
p_{f}, q_{f}
\end{array}\right) ; w_{f}\right\},
$$

where $\mathrm{ff}_{f}\left(\mathbf{X}_{c h}\right)$ defines component $f$ of fitness function $(f=1,2, \ldots, n F F) ; w_{f} \in[0,1]$ stands for component weights; $\operatorname{sig}(\cdot)$ stands for the function used for normalization of components to the range of $[0,1]$; $p_{f}, q_{f}$ are normalization parameters; $T^{*}\{\cdot\}$ is the t-norm with weights of arguments used for aggregation of the fitness function components.

The components of $\mathrm{ff}\left(\mathbf{X}_{c h}\right)$ considered in this paper are shown in Section 4 (including the parameters-see Table 5). The function used to normalize the components (sigmoid) is defined as follows:

$$
\operatorname{sig}(x, p, q)=(1+\exp (p \cdot(q-x)))^{-1},
$$

where $q$ is the center of the acceptable range [xMin, xMax] of the changes of the domain of $x$ parameter (therefore $\operatorname{sig}(q, p, q)=0.5)$ ), and $p$ is used to determine the angle of inclination of the central part of function graph $\operatorname{sig}(\cdot)$ with respect to abscissa. The t-norm with weights of the arguments is defined as follows:

$$
T^{*}\{\mathbf{a} ; \mathbf{w}\}=\prod_{f=1}^{n F F}\left\{1-w_{f} \cdot\left(1-a_{f}\right)\right\}=\prod_{f=1}^{n F F}\left(1-w_{f} \cdot\left(1-a_{f}\right)\right),
$$

where values $w_{f} \in[0,1]$ mean weights of importance of the arguments $a_{f} \in[0,1]$. Please note that $T^{*}\left\{a_{1}, a_{2} ; 1,1\right\}=T\left\{a_{1}, a_{2}\right\}$ and $T^{*}\left\{a_{1}, a_{2} ; 1,0\right\}=a_{1}$.

\begin{tabular}{|c|c|c|c|}
\hline component & definition & description & weights (formula (9)) \\
\hline $\mathrm{ff}_{1}\left(\mathbf{X}_{c h}\right)_{(\mathrm{ACC})}$ & $\sqrt{\frac{\sum_{k=0}^{n I t e r}\left(\begin{array}{c}\left.\omega^{*}(k)+\right)^{2} \\
-\omega(k)\end{array}\right)}{n I t e r}}$ & $\begin{array}{l}\text { difference between set signal } \omega^{*}(t) \text { and } \\
\text { signal } \omega(t)\end{array}$ & $\begin{array}{c}w_{1}=1.00 \\
p_{1}=10.00 \\
q_{1}=0.30\end{array}$ \\
\hline $\mathrm{ff}_{2}\left(\mathbf{X}_{c h}\right)_{(\mathrm{OSC})}$ & $\frac{\sum_{m=1}^{M-1}\left|r_{m}-r_{m+1}\right|}{u \operatorname{Max}-u \operatorname{Min}}$ & $\begin{array}{l}\text { oscillations of a controller expressed } \\
\text { as the sum of the differences between } \\
\text { successive oscillations (Fig. } 5 \text { ) related to } \\
\text { the range }[u M a x, u M i n] \text { of the chances of } \\
\text { controller output } u\end{array}$ & $\begin{array}{l}w_{2}=0.20 \\
p_{2}=1.00 \\
q_{2}=20.00\end{array}$ \\
\hline $\mathrm{ff}_{3}\left(\mathbf{X}_{c h}\right)_{(\mathrm{CMP})}$ & $\frac{\text { Nkeys } \cdot 100 \%}{\text { MaxNkeys }}$ & $\begin{array}{l}\text { complexity of a controller } \\
\text { expressed as a proportion of the } \\
\text { number of active elements to the } \\
\text { maximum number of elements } \\
\text { MaxNkeys }=15 \text { nodes } \cdot 5 \text { keys }=75 \text {, where } \\
15 \text { nodes is a number resulting from the } \\
\text { acceptable height of the treeTreeHeight }\end{array}$ & $\begin{array}{c}w_{3}=0.20 \\
p_{3}=10.00 \\
q_{3}=0.50\end{array}$ \\
\hline $\mathrm{ff}_{4}\left(\mathbf{X}_{c h}\right)_{(\mathrm{NFIL})}$ & Nfilters & number of active filters FIR in PIDC & $\begin{aligned} w_{4} & =0.10 \\
p_{4} & =0.01 \\
q_{4} & =1.50\end{aligned}$ \\
\hline $\mathrm{ff}_{5}\left(\mathbf{X}_{c h}\right)_{(\mathrm{OVH})}$ & $\max _{k=1, \ldots, n \text { Iter }}\left\{\begin{array}{l}\omega(k)- \\
+\omega^{*}(k)\end{array}\right\}$ & $\begin{array}{l}\text { stands for overshooting expressed as } \\
\text { a maximum difference between signal } \\
\omega(k) \text { and desired signal } \omega^{*}(k)\end{array}$ & $\begin{array}{c}w_{5}=0.10 \\
p_{5}=10.00 \\
q_{5}=0.30\end{array}$ \\
\hline
\end{tabular}

\section{Table 5}

The evaluation criteria of fitness function ff $(\cdot)(n F F=5)$ 


\section{Table 6}

Averaged simulation results (each simulation variant was repeated Nrepeats $=40$ times $)$

\begin{tabular}{l|c|c|c|c|c|c}
\hline variants & ACC & OSC & CMP & NFIL & OVH & ff $(\cdot)$ \\
\hline N0+F0 & 0.0684 & 5.9756 & 0.1610 & 0.0000 & 0.0051 & 0.1449 \\
\hline N0+F1 & 0.0684 & 5.9748 & 0.1710 & 1.0250 & 0.0051 & 0.1457 \\
\hline N0+F2 & 0.0684 & 5.9729 & 0.1800 & 3.0000 & 0.0052 & 0.1467 \\
\hline N1+F0 & 0.07776 & 6.6790 & 0.1780 & 0.0000 & 0.0600 & 0.1561 \\
\hline $\mathrm{N} 1+F 1$ & 0.0743 & 7.0941 & 0.1690 & 1.4250 & 0.0739 & 0.1541 \\
\hline $\mathrm{N} 1+\mathrm{F} 2$ & 0.0743 & 6.9947 & 0.1830 & 3.0000 & 0.0743 & 0.1554 \\
\hline $\mathrm{N} 2+\mathrm{F} 0$ & 0.0894 & 4.0407 & 0.1945 & 0.0000 & 0.1029 & 0.1706 \\
\hline $\mathrm{N} 2+\mathrm{F} 1$ & 0.0835 & 4.8588 & 0.1770 & 1.5250 & 0.0889 & 0.1634 \\
\hline $\mathrm{N} 2+\mathrm{F} 2$ & 0.0837 & 4.5800 & 0.1840 & 3.0000 & 0.0884 & 0.1643 \\
\hline
\end{tabular}

Figure 5

The way of determining $r_{m}$ values for function $\mathrm{ff}^{\mathrm{OsC}}(\cdot)$ (see Table 4)

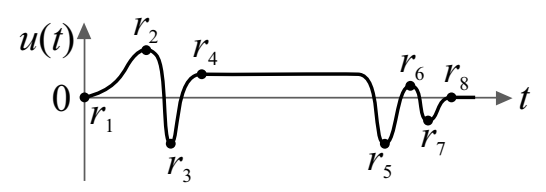

It is worth mentioning that other approaches for criteria aggregation might be also used. However, the approach expressed in formula (9) is simple to implement and easy to expand. In addition, it allows one to give aggregated components a specific validity. This is a significant element for problems with multiple evaluation criteria.

\section{Simulation Results}

Comments on the simulations carried out can be summarized as follows (Fig. 6, Fig. 7).

In the simulations a DC Motor (DCM) problem was considered [11]. This is a control problem in which the goal of the control is to achieve as fast as possible an angular velocity set by the motor shaft $\omega^{*}$. A description of this problem has been shown in Table 2. It is worth noting that the problem considered in the simulations can be seen from a practical point of view as an interesting benchmark in the optimization field.

\section{Figure 6}

Exemplary results obtained for the F1 simulation variant (filters selected dynamically) and different noise values: a) No, b) N1, and c) N2. The dashed line indicates the set signal

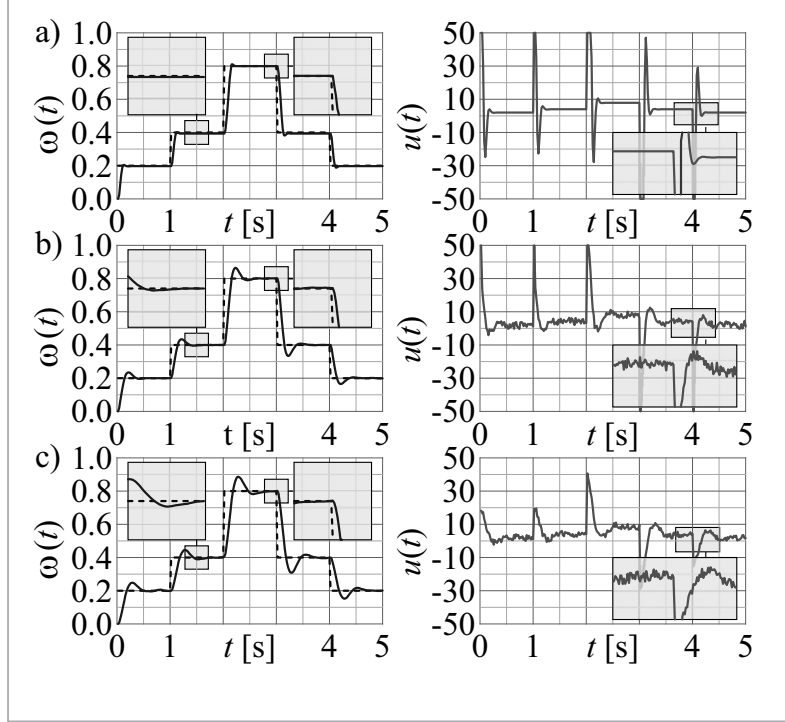

Figure 7

Exemplary results obtained for the F1 simulation variant and verification set signal (different set signal than used in the learning and testing phases) and different noise values: a) N0, b) N1, and c) N2. The dashed line indicates the set signal
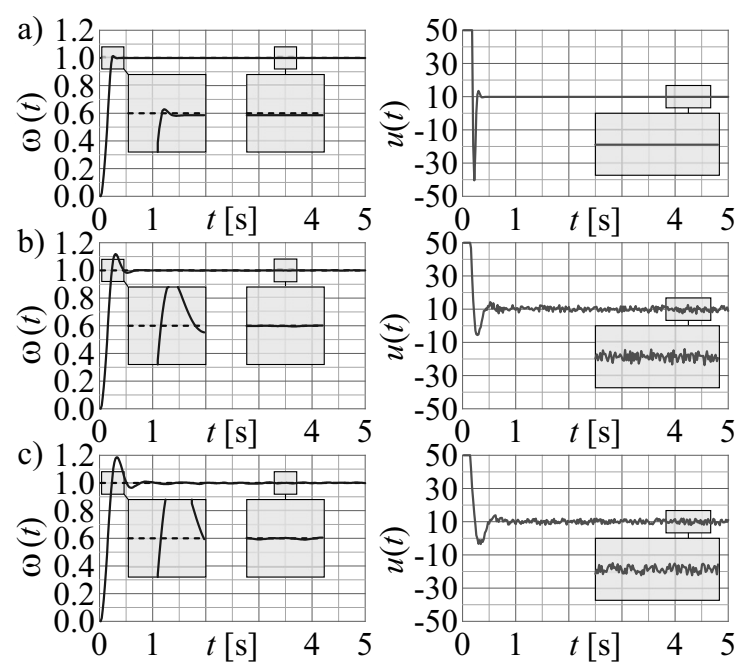
Figure 8

The exemplary results obtained with taking only RMSE as the fitness function for the PIDC for the F1 simulation variant $\left(w^{\mathrm{ACC}}=1.0, w^{\mathrm{OSC}}=0.0, w^{\mathrm{CMP}}=0.0, w^{\mathrm{NFIL}}=0.0\right.$, $w^{\mathrm{OVH}}=0.0$ ) and different noise values: a) No, b) N1, and c) N2. The dashed line indicates the set signal

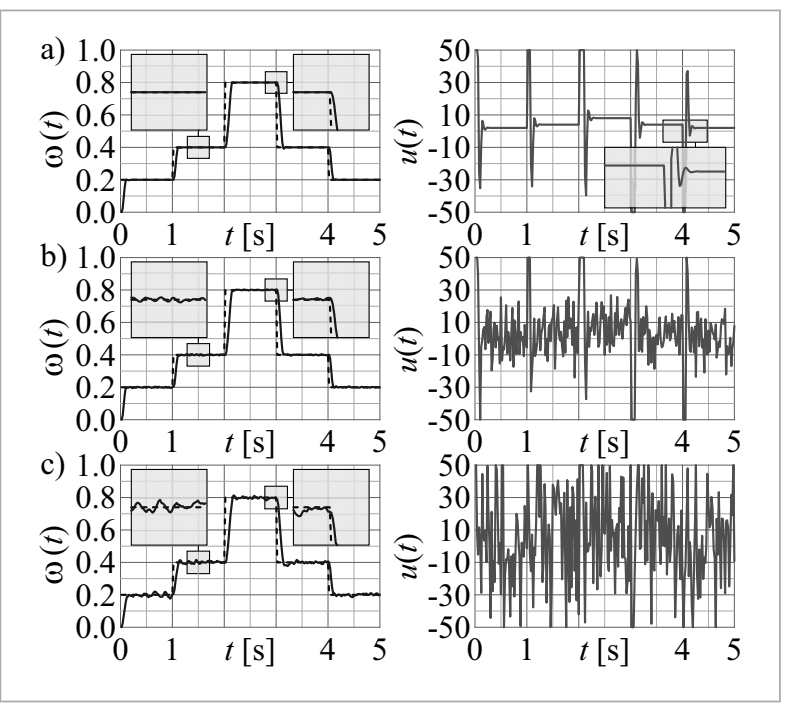

The simulations were performed in different variants in which the assumed noise level of the signals measured from the object varied in the following ways: $0.0 \%$ (variant N0), $0.1 \%$ (variant N1), and $0.5 \%$ (variant N2). The aim of such an approach is to provide simulations with the conditions as close to the real conditions (prevailing in real CSs) as possible. In addition, variants of the PIDC structure, which differed in the way in which the filters were activated: filters not active (variant F0), dynamically activated filters (variant F1), filters active (variant F2) are taken into account.

- In the simulations the GP algorithm was used, whose parameters are shown in Table 4. The purpose of this algorithm was to find the PIDC structure and parameters. The GP algorithm searched for parameter values considering the ranges shown in Table 3.

- In the simulation the solutions (individuals of populations) were evaluated using fitness function ff (.) in the form of (9) with the criteria presented in Table 4. The criteria taken into account include: control error, oscillations (the way in which they are calculated is shown in Fig. 5; it is worth pointing out that the noise is not included-little oscillations are not taken into account), complexity and overshooting. In Table 4 the following additional markings were used: Nkeys that stands for the number of active keys $\left(C_{\mathrm{P}}^{\mathrm{CB}}, C_{\mathrm{I}}^{\mathrm{CB}}\right.$, $C_{\mathrm{D}}^{\mathrm{CB}}, C^{\mathrm{CB}}$ and $C_{l}^{\mathrm{FLL}}$ are treated as keys that allow for reduction of controller elements) in the $\mathrm{CB}$ and $\mathrm{OP}$ blocks; MaxNkeys is the number of active keys in the CB and OP blocks in the PIDC structure with the highest allowable TreeHeight value; Nfilters is the number of active FIR filters in the PIDC structure. In Table 4 weights of criteria $w$ and values of $p, q$ used for criteria normalization by function (10) are given. The values of $p$ and $q$ have been selected in such a way that the function (10) suits/covers in a best way the widest range of possible values of the corresponding criteria.

\section{Figure 9}

The exemplary structures of the PIDC, whose way of peration is shown in Figs 6 and 7

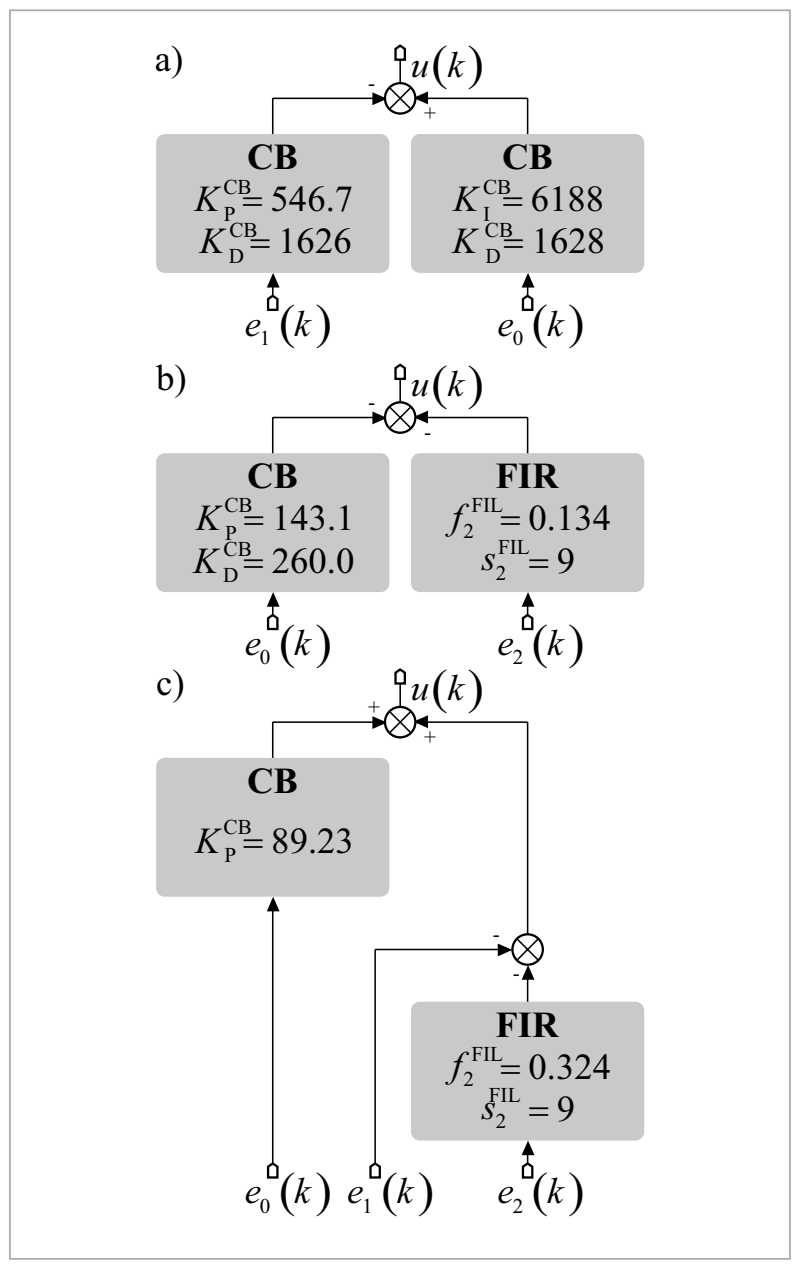


- Each simulation variant has been repeated a certain number of times ( Nrepeats $=40$ ), and the results were averaged (Table 6). Sample waveforms and the corresponding PIDCs structures are shown in Figs 6-9.

The conclusions from the performed simulations can be summed up as follows:

- The results obtained in the simulations are satisfactory. The GP algorithm found a sufficient PIDC structure and parameters for the considered control problem in the sense of the adopted evaluation criteria.

- Taking into account the noise of signals (N1 and N2 variants) coming from the object contributed to the activation of filters in F1 variants (Table 6, column NFIL; Fig. 9). For these variants, the best values of the evaluation function (positive) were obtained (Table 6, column ff $(\cdot)$ ).

- Activation of the filters slightly increased the control accuracy in variants N1 and N2 (Table 6, column ACC). On the other hand, the activation of the filters in variants NO (no noise) caused a slight increase in the value of the evaluation function (Table 6, column $\mathrm{ff}(\cdot)$ ).

- In the simulations, an additional case was considered when only the RMSE was taken into account in the evaluation function $\left(w^{\mathrm{ACC}}=1.0\right.$, $\left.w^{\mathrm{OSC}}=0.0, w^{\mathrm{CMP}}=0.0, w^{\mathrm{NFIL}}=0.0, w^{\mathrm{OVH}}=0.0\right)$. This resulted in a significant increase of oscillations of the control signal, increase in the value of the evaluation function, a reduction in overshooting (this phenomenon is beneficial), and only a slight increase in accuracy compared to the accuracy presented in Table 6. These results were considered as negative and are not included in this article. In Fig. 8 only an example of the result of such selected PIDC is presented.

_ PIDCs designed automatically by the GP work properly also for verification tests with the set signals other than those used in the learning phase (Fig. 7). This is an additional advantage of the approach proposed in this paper.

When comparing the results obtained in this paper with the results obtained by other authors, it is advisable to take into account various assumptions made in the learning phase, various simulation variants and various methods of results presentation (e.g. showing only the best achieved results). This comparison can be summarized as follows:

- The authors in [1] used a different desired signal and the noise of the input signals has not been taken into account. The results obtained for them for neural network with 10 inputs (RMSE $=0.4582)$ are worse than the results obtained in this paper (RMSE=0.1209). At the same time, the results obtained for a neural network with 15 input neurons (RMSE=0.0387) are better than the results obtained in this paper. It is worth noting that in paper [1] an identical testing and verifying set signal was used (in this work different set signals were used), which simplifies the problem under consideration.

_ The authors in [21] used a neural network sliding mode controller and took into account disturbance, load torque and uncertainties of the model with a purpose of improving the control process. The authors in [11] also considered a controller based on a neural network. The presented ideas of using neural networks are interesting, but the interpretation of their mode of operation is difficult. Neural network weights do not have their interpretation, unlike the $\mathrm{P} / \mathrm{I} / \mathrm{D}$ elements used in the PIDC.

- The authors in [6] compared different controllers (PID-, state-space-, cascade ones) and obtained the settling time of the signal at the level of $0.6 \mathrm{~s}$. The settling time for the PIDC selected by the GP was close to $0.35 \mathrm{~s}$ (see Fig. 7.a) and it was obtained for a more complex set signal (in [6] the set signal had a constant value). The authors in [34] also considered the problem of shortening the settling time. They proposed a hybrid-type controller: PID+ANN (artificial neural network) for which the obtained settling time was about $0.5 \mathrm{~s}$.

The authors in [7] considered a controller based on a fuzzy system with 25 fuzzy rules. The obtained settling time was about 3 seconds; however, different parameters for the DCM were set. In addition, a large number of rules may be difficult to interpret. In the literature, descriptions of fuzzy controllers with even more fuzzy rules can be found [8]. In paper [29], we considered a fuzzy system controller (FFPIDC) based on a maximum of 5 fuzzy rules. It combines a fuzzy system with $\mathrm{P} / \mathrm{I} / \mathrm{D}$ terms and FIR filters. The results obtained 
for this controller (whose structure was, however, more complex than the PIDC's) were therefore slightly better than those obtained in this paper (the accuracy of the FFPIDC without filters is equal to 0.0710 , and accuracy of the PIDC without filters is equal to $0.0776-8.5 \%$ worse; the accuracy of the FFPIDC with filters is equal to 0.0690, and the accuracy of the PIDC with filters is equal to $0.0743-7.2 \%$ worse). It is worth noting, however, that the FFPIDC worked with about 3 times less overshooting compared to the PIDC (FFPIDC overshooting is about 0.37 , and PIDC overshooting does not exceed 0.1).

- It can be concluded that the solution proposed in this paper works with a good accuracy and it is characterized by simple implementation and a clear structure (this results from an unambiguous use of $\mathrm{P} / \mathrm{I} / \mathrm{D}$ ). In addition, the obtained controllers were resistant to the noise of measuring signals and are able to function in changing operating conditions (see Fig. 7).

\section{Conclusions}

In this paper possibilities of using genetic programming (GP) for solving complex optimization problems was shown. Such complexity results, among others, from the aim of the algorithm, which is selection of structure and tuning parameters (in a typical approach only parameters of a fixed structure are tuned). Due to that the proposed GP has a universal character and can be used e.g. for designing of neural network structures and their weights, selection of fuzzy system structures and tuning parameters of fuzzy rules, selection of evolutionary operators and

\section{References}

1. Aamir, M. On Replacing PID Controller with ANN Controller for DC Motor Position Control. International Journal of Research Studies in Computing, 2013, 2(1), 21-29. https://doi.org/10.5861/ijrsc.2013.236

2. Abdelbari, H., Shafi, K. Learning Structures of Conceptual Models from Observed Dynamics Using Evolutionary Echo State Networks. Journal of Artificial tuning their parameters, selection of a model and tuning its parameters, etc.

Moreover, this paper shows that the GP algorithm is a very flexible method. It allows for a simple adjustment of the algorithm, its operators and the method used for evaluating individuals to a given problem. What is more, a method of combining the GP with another algorithm, i.e. the genetic algorithm, has been presented. Thanks to that, the GP algorithm can efficiently process solutions with parameters encoded not only in real number values, but also in integral values.

The solutions proposed in this paper have an interdisciplinary character, because they concern the theory of population algorithms and the control theory. The detailed purpose of these solutions is to support designers of controllers based on proven and almost perfectly functioning PID controllers. We have proposed a new approach to designing of complex controllers built on the basis of PID controllers with the filtration of signals coming from the object. The purpose of the used filters with a finite impulse response is to improve the properties of those controllers (e.g. by elimination of controller output oscillations). This is particularly important when there is noise in measuring signals coming from a controlled object. The results obtained in the simulations were very satisfactory.

In the future, it is planned to use the proposed algorithm of genetic programming in other application areas related to complex adaptive systems. Moreover, hardware implementation of the proposed controllers and their testing in real control systems is also planned.

\section{Acknowledgments}

The authors would like to thank the reviewers for their very helpful suggestions and comments in the revision process.

Intelligence and Soft Computing Research, 2018, 8(2), 133-154. https://doi.org/10.1515/jaiscr-2018-0010

3. Agharkakli, A., Sabet, G. S., Barouz, A. Simulation and Analysis of Passive and Active Suspension System Using Quarter Car Model for Different Road Profile. International Journal of Engineering Trends and Technology, 2012, 3(5), 636-644. 
4. Alia, M. A. K., Younes, T. M., Alsabbah, S. A. A Design of a PID Self-Tuning Controller Using LabVIEW. Journal of Software Engineering and Applications, 2011, 4, 161171.https://doi.org/10.4236/jsea.2011.43018

5. Åström, K., Hägglund, T. Automatic Tuning of Simple Regulators with Specifications on Phase and Amplitude Margins. Automatica, 1984, 20(5), 645-651. https://doi. org/10.1016/0005-1098(84)90014-1

6. Baćac, N., Slukić, V., Puškarić, M., Štih, B., Kamenar, E., Zelenika, S. Comparison of Different DC Motor Positioning Control Algorithms. Proceedings of the MIPRO 2014, 2014, 26-30. https://doi.org/10.1109/MIPRO.2014.6859832

7. Bansal, U. K., Narvey, R. Speed Control of DC Motor Using Fuzzy PID Controller. Advance in Electronic and Electric Engineering, 2013, 3(9), 1209-1220.

8. Bature, A. A., Muhammad, M., Abdullahi, A. M. Position Control of a DC Motor: An Experimental Comparative Assessment Between Fuzzy and State Feedback Controller. ARPN Journal of Engineering and Applied Sciences, 2013, 8(12), 984-987.

9. Caraveo, C., Valdez, F., Castillo, O. Optimization of Fuzzy Controller Design Using a New Bee Colony Algorithm with Fuzzy Dynamic Parameter Adaptation. Applied Soft Computing, 2016, 43, 131-142. https://doi. org/10.1016/j.asoc.2016.02.033

10. Chandra A., Chattopadhyay, S. Design of Hardware Efficient FIR FILTER: A REVIEW of the State-of-the-Art Approaches. Engineering Science and Technology, an International Journal, 2016, 19, 212-226.

11. Cheon, K., Kim, J., Haamadache, M., Lee, D. On Replacing PID Controller with Deep Learning Controller for DC Motor System. Journal of Automation and Control Engineering, 2015, 3(6). https://doi.org/10.12720/joace.3.6.452-456

12. Choi, H. H., Jung, J.-W. Discrete-Time Fuzzy Speed Regulator Design for PM Synchronous Motor. IEEE Transactions on Industrial Electronics, 2013, 60(2), 600-607. https://doi.org/10.1109/TIE.2012.2205361

13. Choi, H. H., Yun, H. M., Kim, Y. Implementation of Evolutionary Fuzzy PID Speed Controller for PM Synchronous Motor. IEEE Transactions on Industrial Informatics, 2015, 11(2), 540-547. https://doi.org/10.1109/ TII.2013.2284561

14. Cpałka, K. Design of Interpretable Fuzzy Systems. Springer, 2017. https://doi.org/10.1007/978-3-319-52881-6

15. Cpałka, K., Łapa, K., Przybył, A. A New Approach to Design of Control Systems Using Genetic Programming.
Information Technology and Control, 2015, 44(4), 433442. https://doi.org/10.5755/j01.itc.44.4.10214

16. Das, S., Pan, I., Das, S., Gupta, A. Genetic Algorithm Based Improved Sub-Optimal Model Reduction in Nyquist Plane for Optimal Tuning Rule Extraction of PID and Controllers via Genetic Programming. Proceedings of the 2011 International Conference on Process Automation, Control and Computing, PACC 2011, 2011, 5978962. https://doi.org/10.1109/PACC.2011.5978962

17. Dinesh, P. S., Manikandan, M. Survey on Reconfigurable Fir Filter Architecture. Proceedings of the 2017 Fourth International Conference on Signal Processing, Communication and Networking (ICSCN), Chennai, 2017, 1-3. https://doi.org/10.1109/ICSCN.2017.8085685

18. Do, T. D., Choi, H. H., Jung, J.-W. SDRE-Based Near Optimal Control System Design for PM Synchronous Motor. IEEE Transactions on Industrial Electronics, 2012, 59(11), 4063-4074.https://doi.org/10.1109/ TIE.2011.2174540

19. Duan, X. G., Deng, H., Li, H. X. A Saturation-Based Tuning Method for Fuzzy PID Controller. IEEE Transactions on Industrial Electronics, 2013, 60(11), 5177-5185. https://doi.org/10.1109/TIE.2012.2222858

20. El-Sousy, F. F. M. Intelligent Optimal Recurrent Wavelet Elman Neural Network Control System for Permanent-Magnet Synchronous Motor Servo Derive. IEEE Transactions on Industrial Informatics, 2013, 9(4), 1986-2003. https://doi.org/10.1109/TII.2012.2230638

21. Fallahi, M., Azadi, S. Adaptive Control of a DC Motor Using Neural Network Sliding Mode Control. Proceedings of the International Multi Conference of Engineers and Computer Scientists (IMECS 2009), 2009, 2.

22. Gil, P., Lucena, C., Cardoso, A., Palma, L. B. Gain Tuning of Fuzzy PID Controllers for MIMO Systems: A Performance-Driven Approach. IEEE Transactions on Fuzzy Systems, 2015, 23(4), 757-768. https://doi.org/10.1109/ TFUZZ.2014.2327990

23. Greensted, A. FIR Filters by Windowing. The Lab Book Pages: An Online Collection of Electronics Information, available online: http://www.labbookpages.co.uk/ audio/firWindowing.html, 2010.

24. Jezernik, K., Horvat, R., Curkovic, M.ASwitching Control Strategy for the Reduction of Torque Ripple for PMSM. IEEE Transactions on Industrial Informatics, 2013, 9(3), 1272-1279. https://doi.org/10.1109/TII.2012.2222037

25. Kadlic, B., Sekaj, I., Pernecka, D. Design of Continuous-Time Controllers Using Cartesian Genetic 
Programming. Proceedings of the 19th World Congress, The International Federation of Automatic Control Cape Town, South Africa, 2014. https://doi. org/10.3182/20140824-6-ZA-1003.00915

26. Ko, C. N., Wu, C. J. A PSO-Tuning Method for Design of Fuzzy PID Controllers. Journal of Vibration and Control OnlineFirst, 2007, 14, 1-21.

27. Lai, J. G., Zhou, H., Hu, W. S. A New Adaptive Fuzzy PID Control Method and Its Application in FCBTM. International Journal of Computers Communications \& Control, 2016, 11(3), 394-404. https://doi.org/10.15837/ ijecc.2016.3.753

28. Eapa, K., Cpałka, K. On the Application of a Hybrid Genetic-Firework Algorithm for Controllers Structure and Parameters Selection. Advances in Intelligent Systems and Computing, Springer, 2016, 429, 111-123. https://doi.org/10.1007/978-3-319-28555-9_10

29. Łapa, K., Cpałka, K. Flexible Fuzzy PID Controller (FFPIDC) and a Nature-Inspired Method for Its Construction. IEEE Transactions on Industrial Informatics, 2018, 14, 1078-1088. https://doi.org/10.1109/ TII.2017.2771953

30. Łapa, K., Cpałka, K., Przybył, A., Saito, T. Fuzzy PID Controllers with FIR Filtering and a Method for Their Construction. LNCS, Springer, 2017, 10245, 761-772. https://doi.org/10.1007/978-3-319-59060-8_27

31. Eapa, K., Cpałka, K., Wang, L. New Method for Design of Fuzzy Systems for Nonlinear Modelling Using Different Criteria of Interpretability. LNCS, Springer, 2014, 8467, 212-227. https://doi.org/10.1007/978-3-319-07173-2_20

32. Lin, C.-K., Liu, T.-H., Yang, S.-H. Nonlinear Position Controller Design with Input-Output Linearisation Technique for an Interior Permanent Magnet Synchronous Motor Control System. IET Power Electronics, 2008, 1(1), 14-26. https://doi.org/10.1049/iet-pel:200701777

33. Liu, G., Chen, L., Zhao, W., Jiang, Y., Qu, L. Internal Model Control of Permanent Magnet Synchronized Motor Using SVM Generalized Inverse. IEEE Transactions on Industrial Informatics, 2013, 9(2), 890-898. https://doi. org/10.1109/TII.2012.2222652

34. Madheswaran, M., Muruganandam, M., Simulation and Implementation of PID-ANN Controller for Chopper Fed Embedded PMDC Motor. ICTACT Journal on Soft Computing, 2012, 2(3), 319-324. https://doi. org/10.21917/ijsc.2012.0049

35. Maher, R. A., Mohamed, M. J., An Enhanced Genetic Programming Algorithm for Optimal Controller Design, Intelligent Control and Automation, vol. 4, pp. 94-101, 2013.
36. Malhotra, R., Sodh, R. Boiler Flow Control Using PID and Fuzzy Logic Controller. Proceedings of the IJCSET, 2011, 1(6), 315-31.

37. Morawiec, M. The Adaptive Backstepping Control of Permanent Magnet Synchronous Motor Supplied by Current Source Inverter. IEEE Transactions on Industrial Informatics, 2013, 9(2), 1047-1055. https://doi. org/10.1109/TII.2012.2223478

38. NagaJyothi, G., SriDevi, S. Distributed Arithmetic Architectures for FIR Filters-A Comparative Review. Proceedings of the 2017 International Conference on Wireless Communications, Signal Processing and Networking (WiSPNET), Chennai, 2017, 2684-2690.

39. Perng, J.-W., Chen, G.-Y., Hsieh, S.-C. Optimal PID Controller Design Based on PSO-RBFNN for Wind Turbine Systems. Energies, 2014, 7, 191-209. https://doi. org/10.3390/en'7010191

40. Petkovic, D., Pavlovic, N. D., Cojbašic, Z., Pavlovic, N. T. Adaptive Neuro-Fuzzy Estimation of Underactuated Robotic Gripper Contact Forces. Expert Systems with Applications, 2013, 40, 281-286. https://doi. org/10.1016/j.eswa.2012.07.076

41. Preindl, M., Bolognani, S. Model Predictive Direct Speed Control with Finite Control Set of PMSM Drive Systems. IEEE Transactions on Power Electronics, 2013, 28(2), 1007-1015. https://doi.org/10.1109/TPEL.2012.2204277

42. Rutkowski, L. Computational Intelligence, Springer, 2008. https://doi.org/10.1007/978-3-540-76288-1

43. Sadiqbatcha, S., Jafarzadeh, S., Ampatzidis, Y. Particle Swarm Optimization for Solving a Class of Type-1 And Type-2 Fuzzy Nonlinear Equations. Journal of Artificial Intelligence and Soft Computing Research, 2018, 8(2), 103-110. https://doi.org/10.1515/jaiscr-2018-0007

44. Sandhiya, V., Karthick, S., Valarmathy, M. A Survey of New Reconfigurable Architectures for Implementing FIR Filters with Low Complexity. Proceedings of the 2014 International Conference on Computer Communication and Informatics, Coimbatore, 2014, 1-9. https://doi.org/10.1109/ICCCI.2014.6921804

45. Segovia, R. V., Hsgglund, T., Astrom, K. J. Noise Filtering in PI and PID Control. Proceedings of the American Control Conference, 2013, 1763-1770. https://doi. org/10.1109/ACC.2013.6580091

46. Soltoggio, A. A Comparison of Genetic Programming and Genetic Algorithms in the Design of a Robust, Saturated Control System. Proceedings of the Genetic and Evolutionary Computation-GECCO 2004. Lecture Notes in Computer Science, 2004, 3103, 174-185. 
47. Szczypta, J., Przybył, A., Cpałka, K. Some Aspects of Evolutionary Designing Optimal Controllers. Artificial Intelligence and Soft Computing. LNCS, Springer, 2013, 7895, 91-100.

48. Tai, K., El-Sayed, A. R., Biglarbegian, M., Gonzalez, C. I., Castillo, O., Mahmud, S. Review of Recent Type-2 Fuzzy Controller Applications. Algorithms, 2016, 9(2). https:// doi.org/10.3390/a9020039

49. Tambouratzis, G. Using Particle Swarm Optimization to Accurately Identify Syntactic Phrases in Free Text. Journal of Artificial Intelligence and Soft Computing
Research, 2018, 8(1), 63-67. https://doi.org/10.1515/ jaiscr-2018-0004

50. Tao, C. W., Taur, J. S., Chang, Y. H., Chang, C. W. A Novel Fuzzy-Sliding and Fuzzy-Integral-Sliding Controller for the Twin-Rotor Multi-Input-Multi-Output System. IEEE Transactions on Fuzzy Systems, 2010, 18(5), 893905. https://doi.org/10.1109/TFUZZ.2010.2051447

51. Yang, X.-S. Nature-Inspired Optimization Algorithms. Elsevier, 2016.

52. Ziegler, J., Nichols, N. Optimum Settings for Automatic Controllers. Transactions ASME, 1942, 64(11), 759-765. 